A full characterization of all deterministic dominant strategy incentive compatible, ex-post individually rational, and ex-post budget balanced direct mechanisms in the public good provision problem with independent private values

Christoph Kuzmics

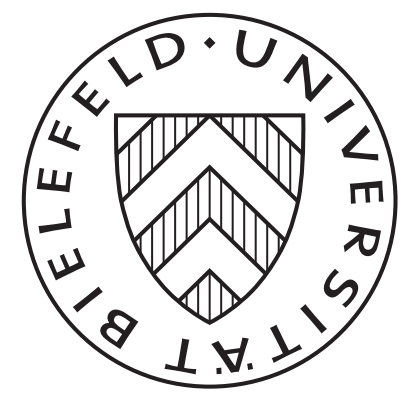




\title{
A full characterization of all deterministic dominant strategy incentive compatible, ex-post individually rational, and ex-post budget balanced direct mechanisms in the public good provision problem with independent private values
}

\author{
Christoph Kuzmics*
}

March 25, 2014

\begin{abstract}
In this note I give a full characterization of all deterministic direct mechanisms in the public good provision problem with independent private values that are dominant strategy incentive compatible, ex-post individually rational, and ex-post budget balanced.
\end{abstract}

Keywords: public good provision, asymmetric information, dominant strategy JEL codes: C72, D82, H41

\section{Introduction}

In this note I give a full characterization of all deterministic direct mechanisms in the public good provision problem with independent private values that are dominant strategy incentive compatible, ex-post individually rational, and ex-post budget balanced.

\section{Setup}

The following is as in Börgers (2013), a special case of the more general d'Aspremont and Gerard-Varet (1979).

A public good problem with independent private values is a tuple consisting of the following ingredients: A set $I$ of $N$ agents; for each agent $i \in I$, a set of possible private

${ }^{*}$ Center for Mathematical Economics, Bielefeld University, christoph.kuzmics@uni-bielefeld.de. I am grateful to Tilman Börgers for writing his book on Mechanism Design, allowing me to teach at Bielefeld University from the manuscript, and for helpful comments and suggestions. 
values (for the indivisible non-excludable public good) $\theta_{i} \in \Theta_{i}=\left[\underline{\theta}_{i} ; \bar{\theta}_{i}\right] \subset[0, \infty$ ), which is private information to the agent; the cost of providing the public good $c>0$. Let $\Theta=\times_{i \in I} \Theta_{i}$ and, for all $i \in I$ let $\Theta_{-i}=\times_{j \in I, j \neq i} \Theta_{j}$ with typical element $\theta_{-i}$.

For a public good problem an allocation rule can be written as a function $q$ from the set of value-profiles, $\Theta$, to the set $\{0,1\}$, where a 1 indicates the provision of the public good and a 0 indicates that the public good is not provided.

A direct mechanism for a public good problem consists of an allocation rule and a set of transfer functions, $t_{i}$, one for each agent $i \in I$, where the transfer (possibly negative) is a money amount that is taken from the agent and given to the mechanism designer. The transfer functions are functions from the set of value-profiles to $\mathbb{R}^{N}$. A direct mechanism for a public good problem is ex-post budget balanced (EPBB) if, for all value-profiles, the sum of all transfers to the designer is equal to the cost of providing the public good if the public good is provided and equal to zero otherwise.

A direct mechanism is dominant strategy incentive compatible (DSIC) if "truth-telling" (i.e. stating ones type) is a (weakly) dominant strategy. It is ex-post individually rational (EPIR) if, for any value-profile, any agent expects a weakly higher payoff from participating in the mechanism than from not participating.

\section{Useful Known Results}

The following is, almost verbatim, Proposition 4.5 of Börgers (2013).

Proposition 1 A direct mechanism is dominant strategy incentive compatible (DSIC) if and only for every $i \in I$ and for every $\theta_{-i} \in \Theta_{-i}$, there are functions $\hat{\theta}_{i}, \tau_{i}$ and $\hat{\tau}_{i}$ from the set $\Theta_{-i}$ to the set of real numbers $\mathbb{R}$ such that:

$$
\begin{aligned}
\theta_{i}<\hat{\theta}_{i}\left(\theta_{-i}\right) & \Rightarrow q\left(\theta_{i}, \theta_{-i}\right)=0 \text { and } t_{i}\left(\theta_{i}, \theta_{-i}\right)=\tau_{i}\left(\theta_{-i}\right) ; \\
\theta_{i}>\hat{\theta}_{i}\left(\theta_{-i}\right) \Rightarrow & q\left(\theta_{i}, \theta_{-i}\right)=1 \text { and } t_{i}\left(\theta_{i}, \theta_{-i}\right)=\hat{\tau}_{i}\left(\theta_{-i}\right) ; \\
\theta_{i}=\hat{\theta}_{i}\left(\theta_{-i}\right) \Rightarrow & q\left(\theta_{i}, \theta_{-i}\right)=0 \text { and } t_{i}\left(\theta_{i}, \theta_{-i}\right)=\tau_{i}\left(\theta_{-i}\right) \text { or } \\
& q\left(\theta_{i}, \theta_{-i}\right)=1 \text { and } t_{i}\left(\theta_{i}, \theta_{-i}\right)=\hat{\tau}_{i}\left(\theta_{-i}\right) ; \\
\hat{\tau}_{i}\left(\theta_{-i}\right)-\tau_{i}\left(\theta_{-i}\right)= & \hat{\theta}_{i}\left(\theta_{-i}\right)
\end{aligned}
$$

The proof is in Börgers (2013).

The following is, almost verbatim, Proposition 4.6 of Börgers (2013).

Proposition 2 A dominant strategy incentive compatible direct mechanism is ex post individually rational (EPIR) if and only for every $i \in I$ and for every $\theta_{-i} \in \Theta_{-i}$ :

$$
t_{i}\left(\underline{\theta}_{i}, \theta_{-i}\right) \leq \underline{\theta}_{i} q\left(\underline{\theta}_{i}, \theta_{-i}\right)
$$




\section{Full Characterization}

Lemma 1 Consider a dominant strategy incentive compatible (DSIC) direct mechanism. For all $i \in I$, let $\hat{\theta}_{i}$ be defined as in Proposition 1 . Then $\hat{\theta}_{i}$, is a weakly decreasing function in all its arguments (i.e. in all $\theta_{j}$ with $j \neq i$ ).

Proof: W.l.o.g. consider agent 1 and consider an arbitrary profile $\theta_{-1}=\left(\theta_{2}, \ldots, \theta_{N}\right)$. Now let $\theta_{1}=\hat{\theta}_{1}\left(\theta_{-1}\right)$. Then, by definition, we have that $q\left(\theta_{1}^{\prime}, \theta_{-1}\right)=0$ for all $\theta_{1}^{\prime}<\theta_{1}$ and $q\left(\theta_{1}^{\prime}, \theta_{-1}\right)=1$ for all $\theta_{1}^{\prime}>\theta_{1}$. Now assume that $q\left(\theta_{1}, \theta_{-1}\right)=1$. W.l.o.g. consider now agent 2. Let $\tilde{\theta}_{2}>\theta_{2}$ and let $\tilde{\theta}_{-1}=\left(\tilde{\theta}_{2}, \theta_{3}, \ldots, \theta_{N}\right)$, or in the case of $N=2$ simply $\tilde{\theta}_{-1}=\tilde{\theta}_{2}$. By DSIC (Proposition 1) for agent 2 we must have that $q\left(\theta_{1}, \tilde{\theta}_{-1}\right)=1$ also. By DSIC (Proposition 1) for agent 1 we then obtain that for all $\theta_{1}^{\prime}>\theta_{1}$ we must have that $q\left(\theta_{1}^{\prime}, \tilde{\theta}_{-1}\right)=1$ as well. Thus, $\hat{\theta}_{1}\left(\theta_{2}^{\prime}, \tilde{\theta}_{-1}\right) \leq \theta_{1}=\hat{\theta}_{1}\left(\theta_{-1}\right)$, which is what we wanted to show.

For the case that $q\left(\theta_{1}, \theta_{-1}\right)=0$ a similar argument applies. Instead of $\tilde{\theta}_{2}>\theta_{2}$ we need to choose $\tilde{\theta}_{2}<\theta_{2}$ and then go through the appropriate steps.

QED

Now to the main result, a version of which has been proven for $N=2$ in Börgers (2013, Proposition 4.8).

Proposition 3 Consider a direct mechanism $(q, t)$ with the property that there is a $\theta \in \Theta$ such that $q(\theta)=1$. This mechanism is dominant strategy incentive compatible (DSIC), ex post individually rational (EPIR), and ex post budget balanced (EPBB) if and only if there are payments $\hat{\tau}_{i} \in \mathbb{R}$ with $\sum_{i \in I} \hat{\tau}_{i}=c$ such that $q(\theta)=1$ and $t_{i}(\theta)=\hat{\tau}_{i}$ for all $i \in I$ if $\theta_{i} \geq \hat{\tau}_{i}$ for all $i \in I$, and $q(\theta)=0$ and $t_{i}(\theta)=0$ for all $i \in I$ otherwise.

Proof: It is easy to see that the given mechanisms satisfy DSIC, EPIR, and EPBB. In what follows I prove the reverse.

Let $\theta \in \Theta$ be such that $q(\theta)=0$. Then EPIR (Proposition 2) implies that $t_{i}(\theta) \leq 0$ for all $i \in I$. EPBB implies that $\sum_{i \in I} t_{i}(\theta)=0$. Together this implies that $t_{i}(\theta)=0$ for all $i \in I$.

Together with Proposition 1 this implies that, using the terminology of Proposition 1 . we have that $\hat{\tau}_{i} \equiv \hat{\theta}_{i}$ for all $i \in I$.

Denote by $\bar{\theta}=\left(\bar{\theta}_{1}, \ldots, \bar{\theta}_{N}\right)$ the vector of maximum values. Now suppose first that $q(\theta)=1$ only if $\theta=\bar{\theta}$. Then the result is trivially satisfied.

Thus, suppose that there is a $\theta \in \Theta$ with $\theta \neq \bar{\theta}$ such that $q(\theta)=1$ and let this $\theta$ be otherwise arbitrary. By DSIC (Lemma 1 and the fact that $\left.\hat{\tau}_{i} \equiv \hat{\theta}_{i}\right)$ we have that $\hat{\tau}_{i}\left(\theta_{-i}\right)$ is weakly decreasing in all its arguments.

DSIC (Proposition 1) implies that $q(\bar{\theta})=1$ also. Now EPBB requires that $\sum_{i \in I} \hat{\tau}_{i}\left(\theta_{-i}\right)=$ $\sum_{i \in I} \hat{\tau}_{i}\left(\bar{\theta}_{-i}\right)=c$. But as $\bar{\theta}_{i} \geq \theta_{i}$ for all $i \in I$ and as all functions $\hat{\tau}_{i}$ are weakly decreasing in all its arguments we must have that all $\hat{\tau}_{i}\left(\theta_{-i}\right)=\hat{\tau}_{i}\left(\bar{\theta}_{-i}\right)$. As $\theta$ with $q(\theta)=1$ was chosen arbitrarily, this implies that for any such $\theta$ we must have that $\hat{\tau}_{i}\left(\theta_{-i}\right)=\hat{\tau}_{i}\left(\bar{\theta}_{-i}\right)$. Thus, all payments are equal to the thresholds and all are constant.

QED 


\section{References}

Börgers, T. (2013): "Introduction to the Theory of Mechanism Design," Book Manuscript, Version May 24, 2013.

D'Aspremont, C., and L.-A. Gerard-Varet (1979): "Incentives and incomplete information," Journal of Public Economics, 11, 25-45. 